\title{
Análise dos movimentos oculares durante a leitura de questões matemáticas por alunos de Engenharia que reprovaram em Cálculo Diferencial Integral I
}

\author{
Maria Marilei Soistak Christo ${ }^{1}$ \\ Universidade Tecnológica Federal do Paraná (UTFPR), Ponta Grossa, PR, Brasil \\ Ângela Inês Klein² \\ Centro de Letras e Comunicação, Universidade Federal de Pelotas, Pelotas, RS, Brasil \\ Luis Mauricio Martins de Resende ${ }^{3}$ \\ Pós-Graduação em Ensino de Ciência e Tecnologia, UTFPR, Ponta Grossa, PR, Brasil \\ Romeu Miqueias Szmoski ${ }^{4}$ \\ Pós-Graduação em Ensino de Ciência e Tecnologia, UTFPR, Ponta Grossa, PR, Brasil \\ Rafael Ribaski Borges ${ }^{5}$ \\ Universidade Tecnológica Federal do Paraná, Ponta Grossa, PR, Brasil
}

Dominik Grätz ${ }^{6}$

Universidade de Potsdam, Potsdam, BB, Alemanha

Resumo: Nesta pesquisa, foram analisados os movimentos oculares de estudantes de Engenharia da UTFPR durante a leitura de questões matemáticas em frente a um rastreador ocular. Participaram da primeira fase 47 alunos que finalizavam a disciplina de Cálculo Diferencial Integral I. Na segunda etapa, participaram nove alunos que reprovaram e refizeram o teste após cursarem CDI I novamente. O objetivo foi comparar os movimentos oculares destes nove alunos que reprovaram em Cálculo Diferencial Integral I os quais leram e resolveram as mesmas questões em dois semestres subsequentes. A partir dos resultados, verificou-se que houve aumento no número de acertos, o tempo médio de resolução e a média das fixações diminuíram, assim como a média de sacadas em duas questões. Por meio da análise dos mapas de calor, foi possível verificar que os comportamentos se repetiram nas duas etapas. Desta forma, conclui-se que os alunos que cursavam a disciplina pela segunda vez apresentaram maior familiaridade com os

\footnotetext{
${ }^{1}$ Doutora em Ensino de Ciência e tecnologia (Universidade Tecnológica Federal do Paraná), Pedagoga na UTFPR Ponta Grossa. Orcid: http://orcid.org/0000-0001-7846-3521. E-mail: msoistak@utfpr.edu.br.

2 Doutora em Linguística (Pontifícia Universidade Católica do Rio Grande do Sul), professora no Centro de Letras e Comunicação da Universidade Federal de Pelotas. Orcid: http://orcid.org/0000-0001-6230-7938. Email: angela.ines.klein@hotmail.com.

3 Doutor em Engenharia Mecânica (Universidade Federal de Santa Catarina), Pró-reitor de Graduação e Educação Profissional. Orcid: http://orcid.org/0000-0003-4151-0843. E-mail: Imresende@utfpr.edu.br.

${ }^{4}$ Doutor em Ciências- Física (Universidade Estadual de Ponta Grossa), Docente na UTFPR Ponta Grossa. Orcid: http://orcid.org/0000-0002-0968-7158. E-mail: rmszmoski@gmail.com.

${ }^{5}$ Doutor em Ciências- Física (Universidade Estadual de Ponta Grossa), Docente na UTFPR Ponta Grossa. Orcid: http://orcid.org/0000-0002-2483-7871. E-mail: rafaelrborges@utfpr.edu.br

${ }^{6}$ Mestrado em Psicologia (Universidade de Potsdam), Pesquisador assistente na Universidade de Potsdam. Orcid: http://orcid.org/0000-0001-5402-6271. E-mail: d.graetz@uni-potsdam.de
} 
Análise dos movimentos oculares durante a leitura de questões matemáticas por alunos de Engenharia que reprovaram em Cálculo Diferencial Integral
Maria Marilei Soistak Christo

Ângela Inês Klein

Luis Mauricio Martins de Resende

Romeu Miqueias Szmoski

Rafael Ribaski Borges

Dominik Grätz

termos matemáticos presentes nos problemas, porém, esta familiaridade não resultou em maior entendimento ou compreensão do problema já que o índice de acertos continuou baixo mesmo na repetição do teste.

Palavras-chave: Movimentos oculares; Matemática; Leitura; Reprovação.

Title: Eye movement analysis from the reading of mathematical questions applied to Engineering students who failed to pass Integral Differential Calculus I

Abstract: The objective of the present study was to analyze eye movement behavior of UTFPR Engineering students during reading of mathematical problems using a eye tracker. In the first phase of this study, eye movements of 47 students who completed the Integral Differential Calculus I course (CDI I) were recorded. In the second phase, nine students who failed to pass and had to retake the exam after attending CDI I again were monitored. Eye movements of these nine students at these two times were compared during reading and solving the same questions in two subsequent semesters. While the number of correctly answered questions increased at the second time, the average solving time and the average fixation duration decreased. In two of three questions, the number of saccades decreased at the second time. Employing heat maps, no differences were found in gaze positions at both times. The authors conclude that students are more familiar with the mathematical terms in these tasks at the second time. However, this did not result in a better comprehension of those problems which is supported by the small amount of correct answers in the second exam.

Keywords: Eye movements; Mathematics; Reading; Exam retake/Reexamination.

\section{Introdução}

A matemática é uma disciplina escolar fundamental que muitos alunos consideram difícil e abstrata. No entanto, especialmente nos cursos de Engenharia, os alunos precisam aprender seus conceitos básicos e adquirir habilidades matemáticas, pois as disciplinas básicas matemáticas são exigidas desde o ingresso na Universidade.

Em relação à aprendizagem da matemática, Zarpelon (2017) afirma, em seu estudo de caso, que se percebe grande dificuldade dos alunos na aprovação de disciplinas desta área, apresentando grande índice de reprovação entre os primeiros períodos dos cursos de Engenharia nas universidades. Garzella (2013) indica que o fracasso dos alunos, especialmente na disciplina de Cálculo Integral Diferencial I (CDI I), decorre do modo de organização da disciplina, normalmente rígida e inflexível, assim como das metodologias de trabalho utilizadas pelos docentes. Zarpelon (2016) conclui que o comprometimento acadêmico interfere de modo expressivo no desempenho dos calouros em CDI I.

No âmbito da UTFPR, 47\% dos cursos de graduação ofertados em 2018 são cursos de Engenharia (52 cursos de 110). Ao analisar os dados disponíveis no sistema acadêmico da instituição, os maiores índices de reprovação encontram-se em disciplinas voltadas à área de 
Análise dos movimentos oculares durante a leitura de questões matemáticas por alunos de Engenharia que reprovaram em Cálculo Diferencial Integral
Maria Marilei Soistak Christo

Ângela Inês Klein

Luis Mauricio Martins de Resende

Romeu Miqueias Szmoski

Rafael Ribaski Borges

Dominik Grätz

exatas, como Cálculo, Física, Geometria Analítica e Álgebra Linear.

Essas percepções levam a refletir sobre o que ocorre durante o processo de ensinoaprendizagem da área de exatas e a repensar por que há alunos que aprendem e outros não. Este trabalho está focado na leitura de problemas matemáticos por alunos que cursavam a disciplina de Cálculo Diferencial Integral I (CDI I). A disciplina de CDI I está contemplada em 91 dos 110 cursos de graduação (85\%) ofertados nos 13 Campi da UTFPR (dados de 2018), incluindo nesta busca cursos de licenciatura, tecnologias e engenharias. A tabela 1 apresenta os índices de reprovação nesta disciplina nos cursos de Engenharia nos últimos cinco anos no Campus Ponta Grossa, incluindo as turmas de reofertas (turmas extras disponibilizadas). Nos dados de reprovação, considerou-se "reprovação por nota" e por "frequência".

Tabela 1 - Reprovação em CDI I nos Cursos de Engenharia da UTFPR: Campus Ponta Grossa

\begin{tabular}{llll}
\hline \multirow{2}{*}{ Período } & $\begin{array}{l}\text { Alunos } \\
\text { Matriculados em CDI I }\end{array}$ & $\begin{array}{l}\text { Alunos Reprovados em } \\
\text { CDI I }\end{array}$ & $\begin{array}{l}\text { \% reprovação em } \\
\text { CDI I }\end{array}$ \\
\hline $\mathbf{2 0 1 4 / 0 1}$ & 272 & 174 & $63,97 \%$ \\
$\mathbf{2 0 1 4 / 0 2}$ & 393 & 290 & $\mathbf{7 3 , 7 9 \%}$ \\
$\mathbf{2 0 1 5 / 0 1}$ & 400 & 254 & $63,5 \%$ \\
$\mathbf{2 0 1 5 / 0 2}$ & 392 & 272 & $69,38 \%$ \\
$\mathbf{2 0 1 6 / 0 1}$ & 376 & 153 & $40,69 \%$ \\
$\mathbf{2 0 1 6 / 0 2}$ & 339 & 235 & $69,32 \%$ \\
\hline $\mathbf{2 0 1 7 / 0 1}$ & 303 & 196 & $64,68 \%$ \\
$\mathbf{2 0 1 7 / 0 2}$ & 338 & 204 & $60,35 \%$ \\
\hline $\mathbf{2 0 1 8 / 0 1}$ & 430 & 234 & $54,41 \%$ \\
$\mathbf{2 0 1 8 / 0 2}$ & 394 & 234 & $59,39 \%$ \\
\hline TOTAL & 3637 & $\mathbf{2 2 4 6}$ & $\mathbf{6 1 , 7 5 \%}$ \\
\hline
\end{tabular}

Fonte: elaborado pelos autores com dados do Relatório Analítico de Gestão (UTFPR, 2019).

Observa-se, na tabela 1, altos índices de reprovação, sendo a média de reprovação em CDI I nestes cinco anos de 61,75\%. A alta taxa de reprovação demonstra a dificuldade encontrada pelos alunos no início do curso universitário, visto que normalmente CDI I é uma disciplina que consta no primeiro período de ingresso. Esse índice acaba criando bolsões de alunos que precisam cursar novamente a disciplina.

Oliveira e Raad (2012) afirmam que a reprovação se constitui num problema crônico, uma verdadeira tradição, mesmo com o uso de bons livros, da didática do docente, do aumento do número de aulas e da busca por aulas mais aplicadas. Os autores avaliam que a mudança desta situação se relaciona "com a renovação do corpo docente e a crescente participação de educadores matemáticos nos cursos de formação de professores" 
Análise dos movimentos oculares durante a leitura de questões matemáticas por alunos de Engenharia que reprovaram em Cálculo Diferencial Integral
Maria Marilei Soistak Christo

Ângela Inês Klein Luis Mauricio Martins de Resende Romeu Miqueias Szmoski Rafael Ribaski Borges Dominik Grätz

(OLIVEIRA; RAAD, 2012, p. 125).

Outra situação encontrada nas leituras sobre desempenho matemático diz respeito à insuficiência de conhecimentos necessários na estrutura cognitiva dos alunos para um bom desempenho na disciplina de Matemática, como relata Rehfeldt et al. (2012) e Zarpelon (2017) em seus estudos. Há uma preocupação constante dos professores engajados com a aprendizagem em buscar estratégias para diminuir esses altos índices de reprovação, mas mesmo com os esforços, é uma situação atual vivenciada pela maioria das universidades e seus cursos de engenharia.

A partir desta constatação, foi formulada esta pesquisa com o intuito de comparar os movimentos oculares durante a leitura de questões de matemática de alunos que reprovaram na disciplina de CDI I e cursaram a mesma no semestre subsequente, a fim de analisar se ocorreu ou não alguma variação no processo de leitura destes alunos. Como diagnósticos de análise foram utilizados o tempo médio de leitura, o número de fixações, os mapas de calor, entre outros. Nossos resultados indicam uma pequena variação de alguns destes parâmetros, o que sugere uma postura diferente dos alunos reprovados no que tange a leitura dos problemas matemáticos. Considerando outros trabalhos que analisam o movimento ocular de leitores proficientes, nossos resultados indicam um pequeno avanço dos alunos, porém, outros estudos e novas amostras são necessários para corroborar esta conclusão já que cada indivíduo pode exibir características próprias de leitura/aprendizagem.

\section{Embasamento teórico}

\section{Leitura e movimentos oculares}

Os movimentos oculares mais utilizados em pesquisas vinculadas à aprendizagem são as sacadas progressivas ou regressivas e as fixações. Tanto as sacadas quanto as fixações apresentam especificidades, demonstrando processos cognitivos distintos.

De acordo com Macedo et al. (2007), as fixações são breves períodos durante os quais o olho permanece examinando uma pequena área do estímulo. Em geral, a localização da fixação reflete a atenção enquanto a duração da fixação representa a dificuldade de processamento. Especificamente, a duração da fixação varia de acordo com tipos de informação (por exemplo, texto ou gráfico) e tipos de tarefas (por exemplo, leitura ou resolução de problemas). Além disso, os locais de fixação e duração refletem as estratégias de leitura dos indivíduos e os conhecimentos ou experiências anteriores. Os padrões de 
Análise dos movimentos oculares durante a leitura de questões matemáticas por alunos de Engenharia que reprovaram em Cálculo Diferencial Integral
Maria Marilei Soistak Christo

Ângela Inês Klein

Luis Mauricio Martins de Resende

Romeu Miqueias Szmoski

Rafael Ribaski Borges

Dominik Grätz

fixação exibem também estratégias cognitivas dos indivíduos utilizadas em tarefas orientadas pelos objetivos propostos pelo pesquisador.

A leitura sugere um processo constante e frequente. Por meio do rastreador, sabe-se que as fixações ocorrem sobre algumas palavras do texto, omitindo normalmente palavras curtas, considerando-se leitores proficientes. De acordo com Rayner (1998), são consideradas palavras curtas aquelas com duas ou três letras. Mesmo que nem todas as palavras sejam fixadas, todas são processadas visualmente, pois se as palavras não fixadas fossem excluídas, o aluno não conseguiria compreender a frase.

Nas fixações, a informação visual é analisada a partir na região foveal que envolve aproximadamente $2^{\circ}$ de diâmetro do campo visual, o que corresponde ao dedo polegar à distância de um braço. Ao redor da fóvea está a região parafoveal, que possui uma resolução relativamente alta e abrange um diâmetro aproximado de $3^{\circ}$ do campo visual. A outra região é a periférica, a qual o leitor não fixa diretamente, mas é capaz de perceber, especialmente quando é proficiente na leitura. Desta forma, analisando o campo visual da região foveal, as fixações correspondem ao foco de atenção visual (FINDLAY; WALKER, 2012).

A sacada, em contrapartida, é o movimento que o olho executa entre cada fixação. A função primária da sacada é trazer nova parte do texto para a região foveal. As sacadas podem ser progressivas (na língua portuguesa, movimento à direita) ou regressivas (movimentos sacádicos em sentido oposto ao da leitura). As sacadas duram em média 200 a 250 milissegundos.

As sacadas regressivas ocorrem 10 a 15\% de vezes durante a leitura (RAYNER, 1998), com a função de retomar a palavra para uma nova inspeção. De acordo com Macedo et al. (2007, p. 274), as sacadas curtas numa mesma palavra indicam uma dificuldade "no posicionamento da fixação, enquanto as longas mostram alta taxa no processamento da palavra". Já as sacadas regressivas maiores que dez letras (MACEDO et al., 2007) demonstram um esforço por parte do leitor na compreensão do conteúdo.

Mapas de calor

Ao examinar as fixações, é possível determinar grande quantidade de informações e analisar os dados, a fim de encontrar evidências de padrões visuais específicos. Ainda, com o auxílio de softwares, é possível apresentar graficamente (por meio de mapas) o comportamento de um ou mais pesquisados. Os mapas de calor são representações gráficas ilustrativas que apresentam os resultados.

Um mapa de calor é capaz de fornecer uma visão por meio de imagens estáticas 
Análise dos movimentos oculares durante a leitura de questões matemáticas por alunos de Engenharia que reprovaram em Cálculo Diferencial Integral
Maria Marilei Soistak Christo

Ângela Inês Klein Luis Mauricio Martins de Resende Romeu Miqueias Szmoski Rafael Ribaski Borges Dominik Grätz

geradas pela sobreposição dos mapas individuais de cada participante. Utiliza-se no mapa uma escala de cores para representar intensidade e o número de fixações realizadas, além do tempo de duração em cada área de interesse. Os mapas de calor apresentam a distribuição visual da atenção, representando em vermelho os pontos com maior atenção, seguindo por pontos amarelo e depois verde. Esta última cor representa a área de menor atenção, ou seja, com menos pontos de fixação. O software que gera os mapas de calor chama-se BeGaze, do fabricante SMI.

De forma qualitativa, o mapa de calor evidencia quais elementos da tela estavam sendo observados pelos participantes da pesquisa.

A média de tempo de fixação nos mapas de calor varia de acordo com a tela que foi visualizada e com os participantes, mas, de forma geral, pode-se dizer que são consideradas áreas de interesse aquelas que são fixadas por 140 a 300 m/s (BARRETO, 2012). Os pontos com maior fixação ficam aparentes nos mapas de calor com as referidas cores e tamanhos de acordo com a quantidade do tempo de fixação.

\section{Metodologia}

\section{Amostragem}

Esta pesquisa está constituída de duas etapas:

1) Coleta de dados no início do segundo semestre de 2017 com quarenta e sete alunos de cursos de Engenharia da Universidade Tecnológica Federal do Paraná - Campus Ponta Grossa, que haviam concluído a disciplina de CDI I).

Foram verificados os alunos que reprovaram em CDI I ao final do 10 semestre de 2017 e que cursariam novamente a disciplina no 2ㅇ semestre de 2017. Dos 47 alunos, 19 reprovaram em CDI I e foram convidados a refazer o teste no início do 10 semestre de 2018, após cursarem a disciplina pela segunda vez. Entretanto, apenas nove compareceram na segunda etapa da pesquisa.

2) Reaplicação das questões aos nove alunos que cursaram a disciplina por dois semestres consecutivos no início do semestre de 2018. Destes, seis foram aprovados e três reprovaram novamente pela segunda vez. 
Análise dos movimentos oculares durante a leitura de questões matemáticas por alunos de Engenharia que reprovaram em Cálculo Diferencial Integral
Maria Marilei Soistak Christo

Ângela Inês Klein Luis Mauricio Martins de Resende Romeu Miqueias Szmoski Rafael Ribaski Borges Dominik Grätz

\section{Especificações Técnicas do Aparelho}

Para a coleta de dados, foi utilizado o rastreador ocular RED 500 do fabricante alemão SMI (Senso Motoric Instruments) para o registro dos movimentos oculares. $O$ registro do movimento ocular é feito pelo equipamento por meio de luz infravermelha. A luz reflete na córnea do olho e volta para a tela do computador, onde é registrada a sua posição.

O rastreador ocular estacionário permite a movimentação desprendida da cabeça de $40 \mathrm{~cm}$ na horizontal e $20 \mathrm{~cm}$ na vertical. Há necessidade também de manter uma distância de 60 a $80 \mathrm{~cm}$ entre os olhos e o monitor do computador de 22 polegadas que contém até 9 pontos de calibragem, portanto o aparelho permite movimentação pertinente sem comprometer os resultados.

Este rastreador é considerado rápido, já que realiza uma coleta do movimento do olho a cada 2 milissegundos (m/s), sendo de $500 \mathrm{~Hz}$. Sua resolução espacial é de 0,03‥ 0 rastreador está munido de três softwares: BeGaze, iView $X$ e Experiment Center.

\section{Descrição do Instrumento}

Foram formuladas três questões por um dos professores de CDI I da instituição, visando trabalhar conceitos básicos da disciplina. Elas foram validadas por dez alunos que já tinham cursado a disciplina. Estão descritas a seguir:

1) Dada uma função de uma variável $f(x)$, qual operação matemática é descrita pela análise de comportamento de $f(x)$ ao tomarmos valores de " $x$ " arbitrariamente grandes?

2) Considere uma função $f(t)$ que varia com o tempo, e um momento "a". Qual quantidade matemática é obtida ao calcular o quociente da variação de $f(t)$ pela variação de tempo, tomando como momento inicial "a" e o momento final cada vez mais próximo de "a"?

3) Considere a função $f(x)=x^{3}$ definida no intervalo $[0,1]$. Se dividirmos o intervalo $[0,1]$ em " $n$ " intervalos de comprimento $1 / n$, e considerarmos retângulos com base igual a cada um desses intervalos e altura igual $f(1 / n)$, para qual número se aproxima a soma dessas áreas quando aumentamos o valor de " $n$ "? 
Análise dos movimentos oculares durante a leitura de questões matemáticas por alunos de Engenharia que reprovaram em Cálculo Diferencial Integral
Maria Marilei Soistak Christo

Ângela Inês Klein Luis Mauricio Martins de Resende Romeu Miqueias Szmoski Rafael Ribaski Borges Dominik Grätz

\section{Aplicação do Instrumento}

Foi solicitado aos alunos que lessem as questões individualmente em frente ao monitor e, ao concluírem a leitura, explicassem verbalmente o que entenderam. Na sequência, resolveram as questões no papel, cujo tempo foi cronometrado, pois não há possibilidade de registrar o movimento ocular durante a resolução dos problemas por escrito. O mesmo procedimento foi realizado nos dois semestres consecutivos.

Os participantes indicaram seu aceite por meio de Termo de Consentimento Livre e Esclarecido, seguindo as orientações do Comitê de Ética em Pesquisa da Universidade Tecnológica Federal do Paraná de 22 de junho de 2017, parecer no 2.132.697.

\section{Levantamento e Tabulação dos Dados}

A abordagem ocorreu através das ferramentas da estatística descritiva, sumarizando e descrevendo o percentual (\%) de acerto, tempo médio de resolução, número médio de sacadas e fixações.

Já a abordagem qualitativa foi utilizada por meio dos mapas de calor. Os mapas de calor são uma ferramenta de visualização de dados e são utilizados para identificar o local de concentração de fixações. Os mapas apresentam ainda a distribuição visual da atenção, respeitando a sequência decrescente das cores vermelho-amarelo-verde como áreas de atenção (pontos de fixação).

Optou-se por fazer a análise descritiva dos mapas de calor por considerar ser uma maneira estratégica para verificar a concentração das fixações, pois, de acordo com Duchowski (2007, p. 46), a permanência do olhar numa região, onde a retina se estabiliza, indica um local de interesse.

Para organização dos valores apresentados na tabela 2, utilizou-se a média, somando os dados dos nove alunos em cada um dos semestres e dividindo por 9 . Somente a $\%$ de acerto não realiza a média. 
Análise dos movimentos oculares durante a leitura de questões matemáticas por alunos de Engenharia que reprovaram em Cálculo Diferencial Integral
Maria Marilei Soistak Christo

Ângela Inês Klein

Luis Mauricio Martins de Resende

Romeu Miqueias Szmoski

Rafael Ribaski Borges

Dominik Grätz

Tabela 2 - Situações problema aplicadas na pesquisa CDI I: 9 alunos que fizeram o mesmo teste em dois semestres

\begin{tabular}{|c|c|c|c|c|c|c|c|c|}
\hline № da questão - & 1 & 1 & 2 & 2 & 3 & 3 & Média & Média \\
\hline Semestre & $1 \underline{a}$ & $2^{\mathrm{a}}$ & $1^{\underline{a}}$ & $2 \underline{a}$ & $1^{\underline{a}}$ & $2 \underline{a}$ & 1ㅇosem & 2ㅇsem \\
\hline$\%$ de acerto & $55 \%$ & $66 \%$ & $22 \%$ & $33 \%$ & $22 \%$ & $44 \%$ & $33 \%$ & $47 \%$ \\
\hline $\begin{array}{l}\text { Tempo médio de } \\
\text { resolução (seg.) }\end{array}$ & 142 & 117 & 167 & 101 & 171 & 108 & 160 & 108 \\
\hline Média de sacadas & 255 & 216 & 431 & 436 & 498 & 469 & 394 & 373 \\
\hline Média de fixações & 255 & 201 & 423 & 353 & 472 & 431 & 383 & 328 \\
\hline
\end{tabular}

Fonte: Autoria própria.

\section{Análise e discussão dos dados}

Retomando os dados apresentados na tabela 2, verifica-se que o número de acertos aumentou quando os alunos fizeram o teste pela segunda vez, entretanto o aumento foi pequeno (média geral passou de 33\% para 47\%) considerando que os alunos cursaram novamente a disciplina e que as questões estão relacionadas a conceitos básicos de CDI I.

Em relação ao tempo médio de resolução das questões (em segundos), é possível perceber que, no segundo teste, os alunos precisaram de menos tempo para resolver as três questões. Entretanto, como nenhum dos alunos comentou lembrar das questões reconhecendo-as da etapa anterior não é possível afirmar se lembravam ou não das mesmas, o que leva os pesquisadores a inferir que o menor tempo de resolução ocorreu por terem tido um semestre a mais com a disciplina e maior familiaridade com os termos e palavras.

Essa familiaridade com os termos também se evidenciou no número de sacadas: percebe-se que em duas questões houve uma diminuição da quantidade de sacadas da primeira para a segunda etapa. Segundo Forster (2017), o número de sacadas tende a ser menor em textos com os quais o leitor tenha mais contato.

A média do número de fixações diminuiu de um semestre para outro. Segundo Forster (2017), o número de fixação costuma se associar à reserva de recursos cognitivos e a um aumento da carga de processamento cognitivo, sendo possível inferir que um número maior das fixações estaria associado à maior dificuldade de processamento. Assim é possível considerar que palavras com fixações mais longas sejam mais difíceis de processar. De acordo com esse mesmo autor (FORSTER, 2017), embora seja possível inferir que a maior quantidade de fixações ocorra em palavras menos frequentes na língua e que esse fator acarrete em dificuldade no processo de análise linguística, os alunos, depois de terem 
Análise dos movimentos oculares durante a leitura de questões matemáticas por alunos de Engenharia que reprovaram em Cálculo Diferencial Integral
Maria Marilei Soistak Christo

Ângela Inês Klein Luis Mauricio Martins de Resende Romeu Miqueias Szmoski Rafael Ribaski Borges Dominik Grätz

cursado CDI I pela segunda vez, provavelmente tem mais familiaridade com as sentenças/proposições.

Além dessa análise quantitativa foram realizadas análises qualitativas por meio dos mapas de calor. As Figuras 1, 2 e 3 são os mapas de calor dos nove alunos que participaram do experimento nas duas etapas. Ao lado esquerdo, as fixações das questões dos nove alunos que cursaram CDI I pela primeira vez e ao lado direito as fixações da segunda vez que fizeram o teste. É possível verificar nas figuras 1,2 e 3 alguns comportamentos que se repetem. Por exemplo, na figura 1, nitidamente, os alunos fixam, por mais tempo nos dois testes, o termo $f(x)$, e também no segundo teste observa-se tamanho e intensidade maiores neste termo, sugerindo que ele está focando ou dedicando maior atenção sobre ele. Nota-se um comportamento semelhante nos termos " $x$ " e "grande", os quais foram pouco destacados no primeiro teste, assim como não no segundo, sugerindo uma preocupação maior dos pesquisados em selecionar (filtrar) as informações relevantes para a resolução do problema. Também na Figura 3 pode ser observado maior concentração de fixação (regiões quentes do mapa de calor) em áreas de interesse importantes para resolução do problema como é o caso da função " $f(x)=x^{3}$ ", do intervalo " $1 / n$ " e da consideração de aumentar " $n$ ". Por outro lado, na Figura 2, que trata da questão em que a porcentagem de acertos foi a mais baixa tanto na primeira como na segunda aplicação, algumas informações necessárias para o entendimento e resolução do problema parecem não ter sido consideradas importantes na leitura como, por exemplo, o estabelecimento dos momentos inicial e final próximos de "a". Assim, embora os mapas de calor desta questão demonstrem a grande redução do número de fixações já apresentada na Tabela 2 e, portanto, sugiram maior familiaridade com a linguagem matemática dos alunos (evidenciada, por exemplo, no fato de se ler apenas " $\mathrm{f}(\mathrm{t})$ " na segunda aplicação e subentender que a função varia com o tempo), os alunos não conseguiram situar ou localizar estas poucas fixações sobre as áreas de interesse relevantes para entender e resolver o problema.

Além das áreas de interesse mais fixadas, que são perceptíveis pela cor vermelha intensa, verifica-se que os participantes fixaram seus olhos em outros pontos do estímulo durante a leitura das questões, especialmente elementos textuais e numéricos que indicam que se trata de questões matemáticas. 
Análise dos movimentos oculares durante a leitura de questões matemáticas por alunos de Engenharia que reprovaram em Cálculo Diferencial Integral
Maria Marilei Soistak Christo

Ângela Inês Klein

Luis Mauricio Martins de Resende

Romeu Miqueias Szmoski

Rafael Ribaski Borges

Dominik Grätz

Figura 1 - Questão 1 de CDI I: Fixações na primeira (figura à esquerda) e segunda etapa (figura à direita) de resolução dos 9 alunos

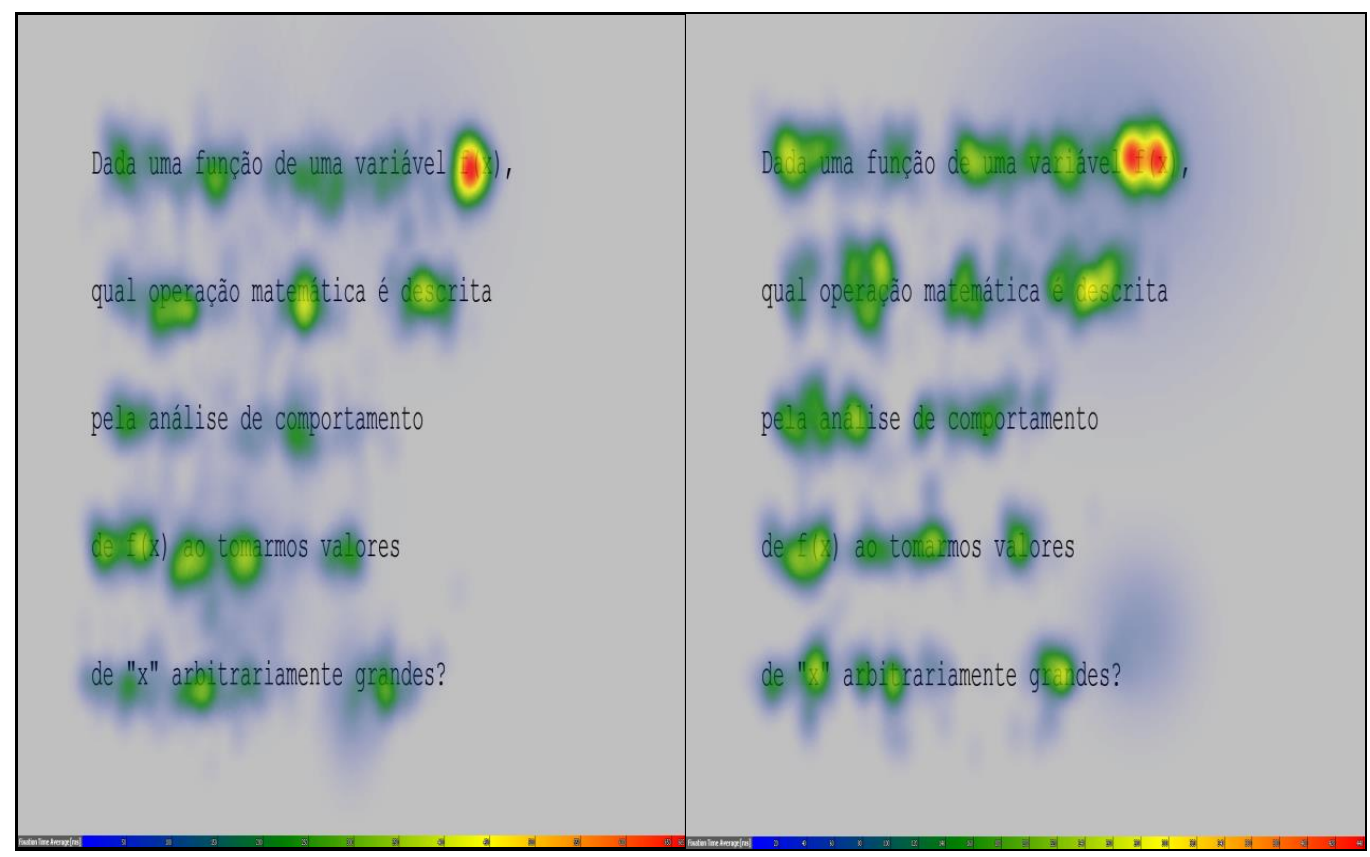

Fonte: Autoria própria.

Figura 2 - Questão 2 de CDI I: Fixações na primeira (figura à esquerda) e segunda etapa (figura à direita) de resolução dos 9 alunos

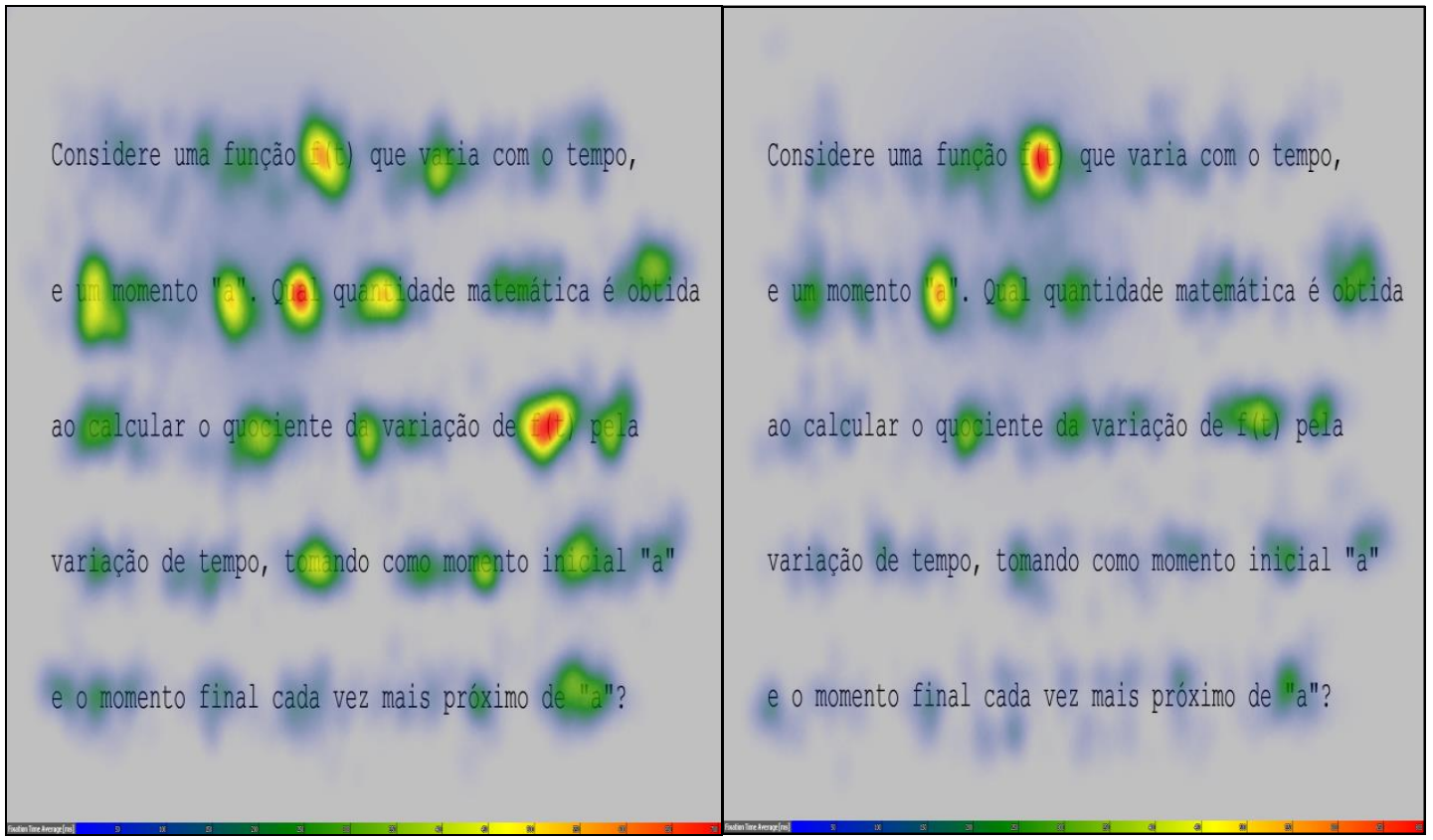

Fonte: Autoria própria. 
Análise dos movimentos oculares durante a leitura de questões matemáticas por alunos de Engenharia que reprovaram em Cálculo Diferencial Integral
Maria Marilei Soistak Christo

Ângela Inês Klein

Luis Mauricio Martins de Resende

Romeu Miqueias Szmoski

Rafael Ribaski Borges

Dominik Grätz

Figura 3 - Questão 3 de CDI I: Fixações na primeira (figura à esquerda) e segunda etapa (figura à direita) de resolução dos 9 alunos

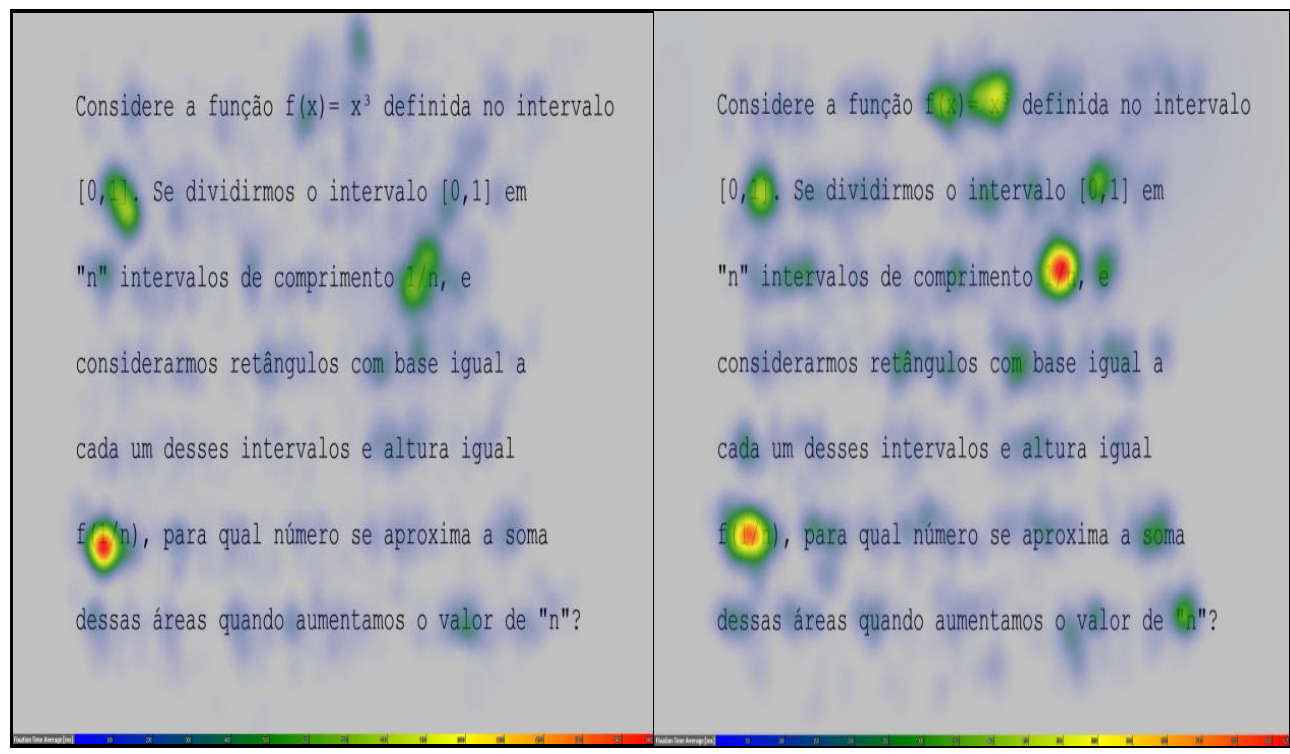

Fonte: Autoria própria.

As figuras 1, 2 e 3, assim como a tabela 2, demonstram que os alunos fizeram mais fixações na primeira vez que realizaram o teste, corroborando com Forster (2017), que diz que quanto mais familiaridade com o assunto lido, menos fixações são realizadas. É possível inferir que houve mais dificuldade de processamento cognitivo quando os alunos resolveram as questões pela primeira vez, conforme os pesquisadores Just e Carpenter (1975), Rayner e Duffy (1986), Inhoff e Rayner (1986) sugerem em seus estudos.

As figuras 4, 5 e 6 apresentam os mapas de calor referentes ao segundo teste realizado pelos nove alunos, considerando quem acertou e quem errou a questão. Ao analisar as Figuras 4 e 6, nota-se maiores quantidades de fixações para aqueles que acertaram em comparação aos que erraram o problema. Considerando a quantidade de fixações um indicativo da familiaridade com os termos matemáticos (linguagem matemática) presente nos problemas, então os resultados sugerem uma distinção entre familiaridade e compreensão, ou seja, uma familiaridade maior com os elementos presentes no texto não implica necessariamente melhor entendimento ou compreensão do mesmo. Mais precisamente, enquanto a Tabela 2 apresenta uma diminuição no número de fixações na segunda leitura, o que, de acordo com a teoria, sugere maior familiaridade com o texto, as Figuras 4 e 6 mostram que aqueles que acertaram realizaram mais fixações do que aqueles que erraram levando, assim, a conclusão de que a familiaridade por si só não garante o entendimento do texto. Isto não quer dizer que um maior entendimento requer mais fixações, pois na Figura 5 os que erraram apresentaram mais fixações do que aqueles que acertaram. Portanto, tais observações permitem inferir apenas que há uma distinção entre 
Análise dos movimentos oculares durante a leitura de questões matemáticas por alunos de Engenharia que reprovaram em Cálculo Diferencial Integral
Maria Marilei Soistak Christo

Ângela Inês Klein Luis Mauricio Martins de Resende

Romeu Miqueias Szmoski

Rafael Ribaski Borges

Dominik Grätz

familiaridade e compreensão e que não a análise apenas das fixações não permite estabelecer um padrão dos que acertaram ou erraram o problema.

Figura 4 - Fixações questão 1 de CDI I: acertos (figura à esquerda) e erros (figura à direita)

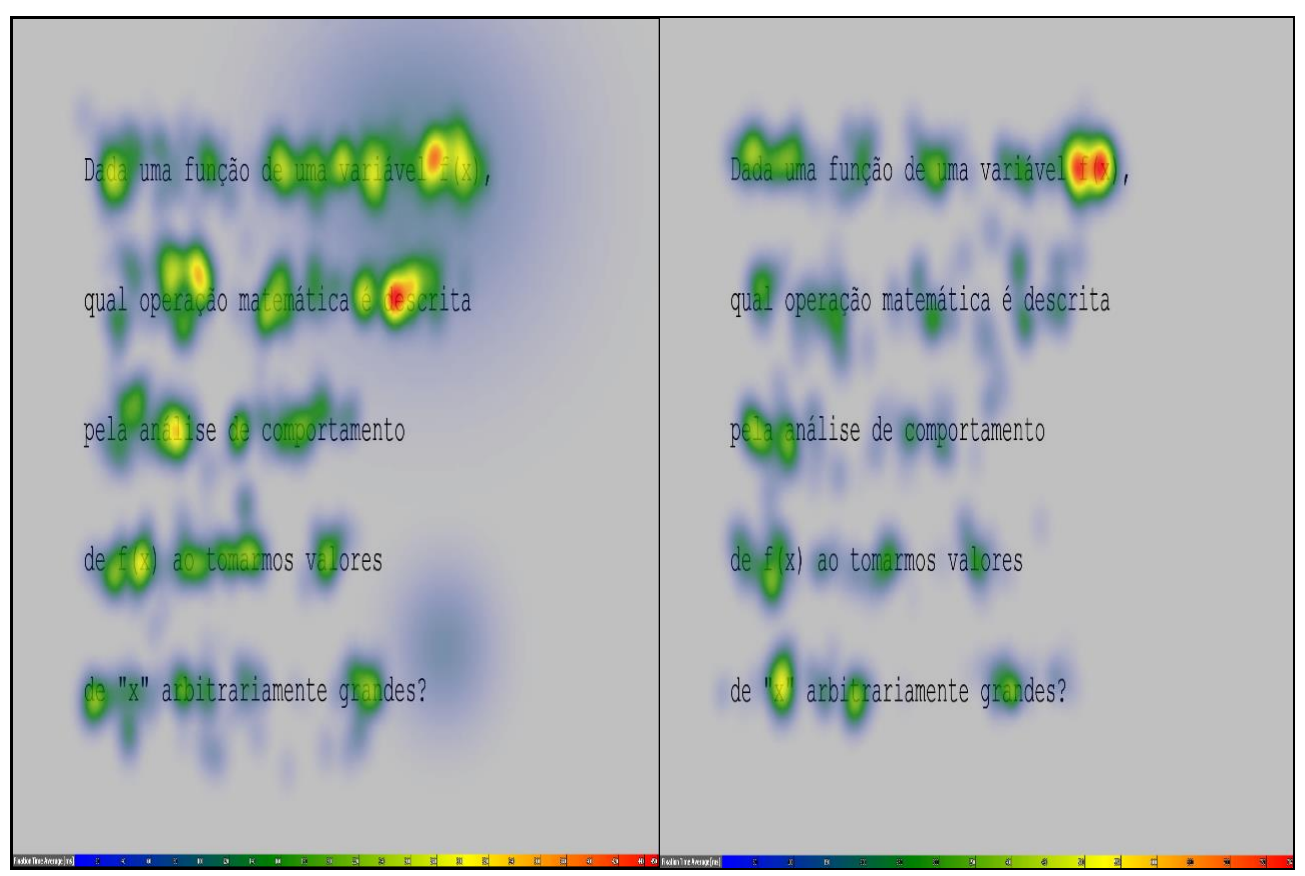

Fonte: Autoria própria.

Figura 5 - Questão 2 de CDI I: acertos (figura à esquerda) e erros (figura à direita)

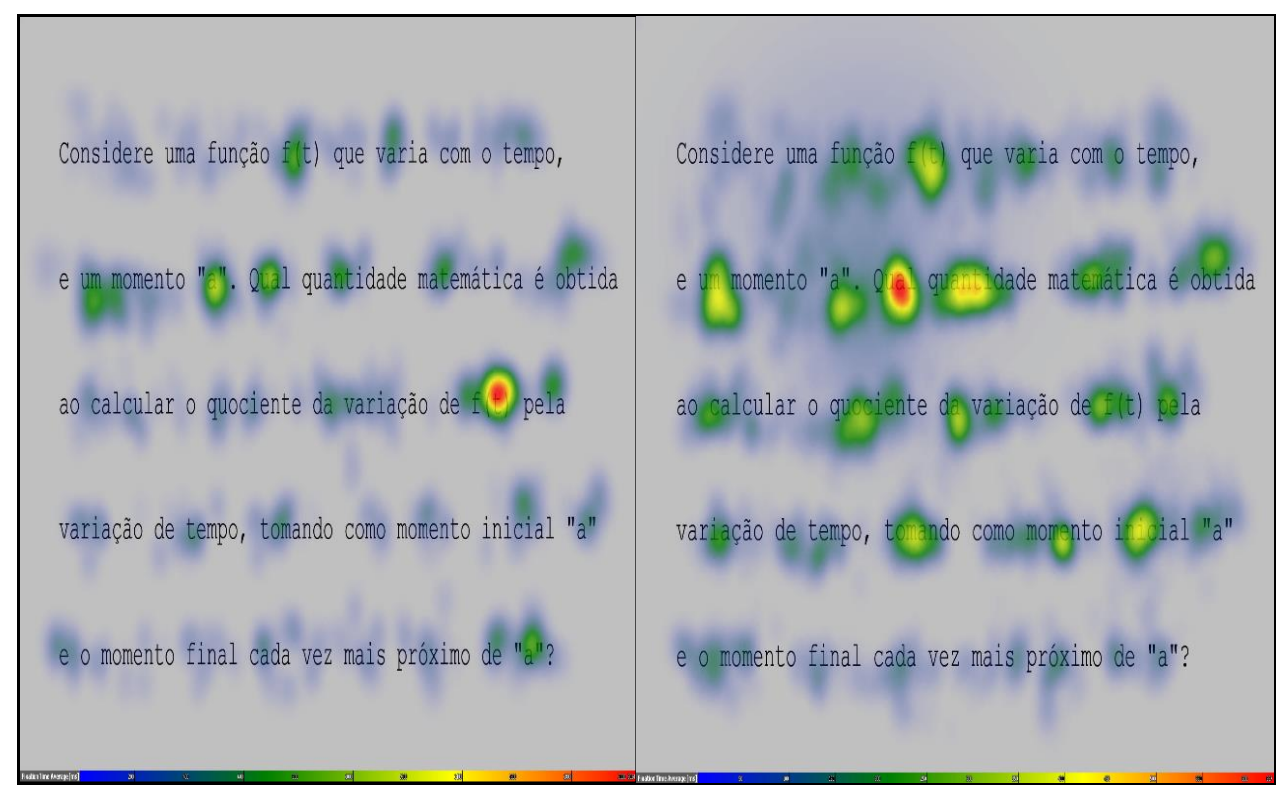

Fonte: Autoria própria. 
Análise dos movimentos oculares durante a leitura de questões matemáticas por alunos de Engenharia que reprovaram em Cálculo Diferencial Integral
Maria Marilei Soistak Christo

Ângela Inês Klein

Luis Mauricio Martins de Resende

Romeu Miqueias Szmoski

Rafael Ribaski Borges

Dominik Grätz

Figura 6 - Questão 3 de CDI I: acertos (figura a esquerda) e erros (figura a direita)

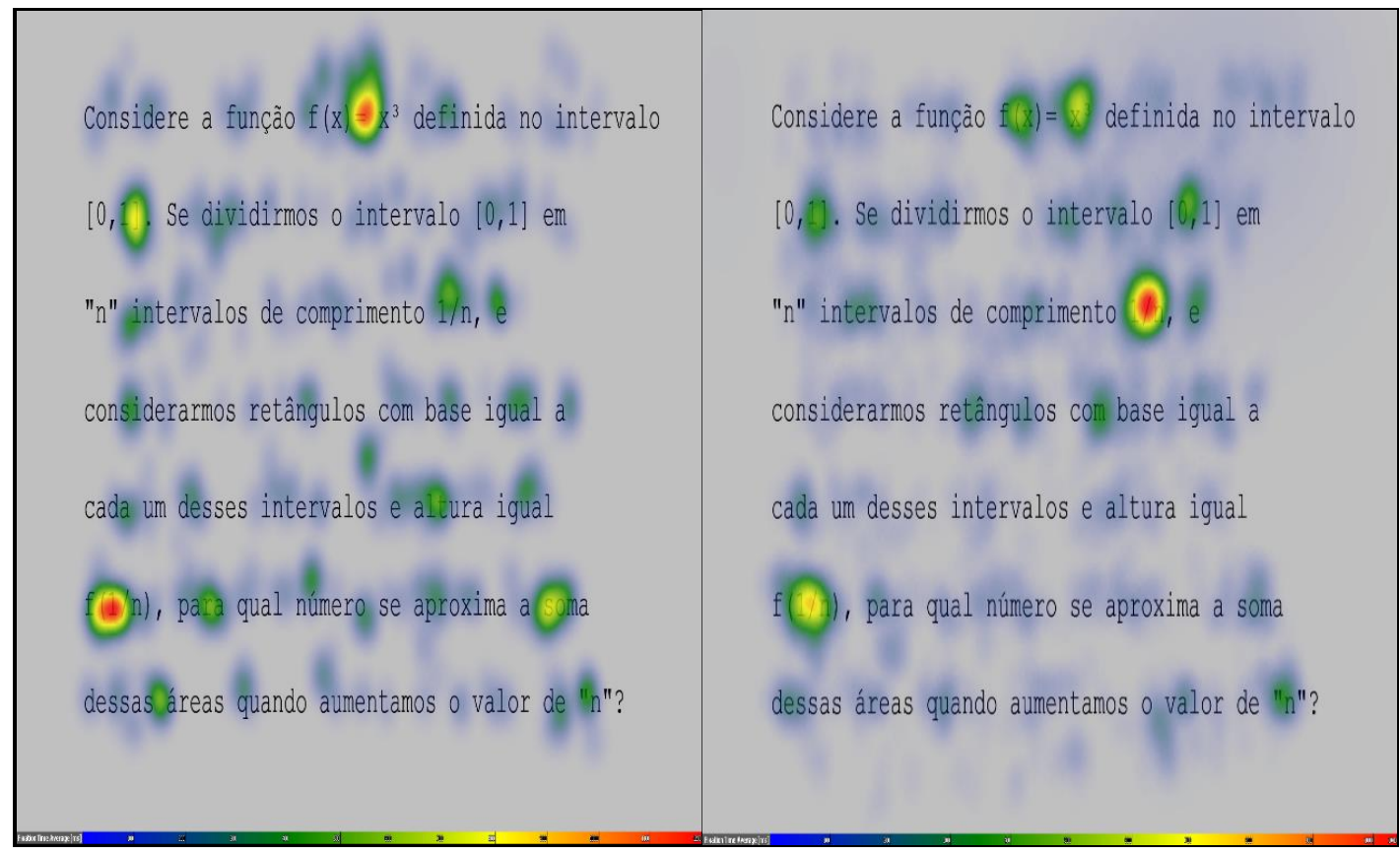

Fonte: Autoria própria.

Como as fixações não foram suficientes para distinguir entre os que acertaram ou erraram os problemas, comparou-se os mapas de calor dos que acertaram e erraram as questões, Figuras 4, 5 e 6, com base na localização das fixações sobre áreas ou informações do texto consideradas mais relevantes para a resolução. Enquanto nas questões 4 e 6 verifica-se uma maior atenção (maior fixação) sobre termos importantes tais como "comportamento de $f(x)$ " (questão 1) e "soma" (questão 3), não identificamos diferenças significativas entre o mapa de calor daqueles que acertaram ou erraram a questão 2 (Figura 5) em relação às áreas de interesse ou pertinentes à resolução. Portanto, também aqui é possível inferir que não há um padrão de leitura, diferenciando os alunos que acertaram a questão dos que erraram. O processamento cognitivo, ilustrado através dos mapas de calor, não evidenciou um comportamento ocular típico de quem acertou ou errou a questão. As figuras 4 e 6 demonstram similaridade ao haver maior número de fixação entre os alunos que acertaram a respectiva questão, tendo consequentemente maior atenção e compreensão da situação problema.

Durante a correção das questões, observou-se que um aluno, na primeira etapa, tinha acertado a questão 1 e errado as questões 2 e 3 . Entretanto, na segunda etapa em que resolveu as mesmas questões, errou a questão 1 e acertou as questões 2 e 3. Christo (2019, p. 70) cita que a "realidade nas universidades demonstra a dificuldade em resolver o 
Análise dos movimentos oculares durante a leitura de questões matemáticas por alunos de Engenharia que reprovaram em Cálculo Diferencial Integral
Maria Marilei Soistak Christo

Ângela Inês Klein

Luis Mauricio Martins de Resende

Romeu Miqueias Szmoski

Rafael Ribaski Borges

Dominik Grätz

problema das reprovações, especialmente nas disciplinas matemáticas". O caso do aluno que trocou os acertos na segunda resolução das questões demonstra a dificuldade encontrada pelos alunos nos conteúdos desenvolvidos em CDI I, que leva a acertos em um semestre e erros em outro na mesma questão. É possível inferir que, com uma amostra maior, talvez esse fato fosse minimizado e surgisse um padrão mais claro nos resultados.

\section{Conclusões}

Esta pesquisa objetivou comparar os movimentos oculares durante a leitura de questões de matemática de alunos que reprovaram na disciplina e cursaram a mesma no semestre subsequente, a fim de tentar compreender o comportamento ocular dos alunos durante a leitura e resolução das mesmas questões por dois semestres diferentes. Pesquisas com o eye tracking são um suporte para este tipo de estudo.

Os movimentos oculares, considerando que através da visão adquire-se conhecimento, são bons aliados para se verificar o processamento cognitivo do aluno, ou seja, como ele está aprendendo. Assim, pesquisas com rastreadores oculares possuem um grande potencial no sentido de auxiliar os professores a perceberem como os alunos processam a informação.

A matemática é uma área que ainda possui poucas pesquisas com o uso do rastreador ocular, mas que pode vir a usufruir com os resultados de pesquisas que utilizam estímulos visuais para análises, ao disponibilizar dados sobre o processamento cognitivo que auxiliam na compreensão da matemática.

Em relação ao trabalho realizado com os alunos de engenharia, é possível concluir que os alunos que reprovaram na disciplina e refizeram o teste não demonstraram grande diferença nas duas etapas. Em relação ao número de acertos, mesmo aumentando na segunda etapa, ainda foi um aumento considerado baixo, visto que houve um semestre inteiro de estudos para melhorar a compreensão do conteúdo da disciplina.

Os mapas de calor demonstram que os pontos mais fixados são parecidos nos dois semestres, embora sacadas e fixações tenham diminuído no segundo semestre. Sobre isso, pode-se inferir que, na segunda etapa, os alunos já tinham maior intimidade com as palavras lidas visto que faziam a disciplina pela segunda vez, pois o número de acertos não mudou consideravelmente devido à diminuição de sacadas e fixações.

Por meio das análises realizadas nesta pesquisa, não foi possível definir um padrão de leitura entre os grupos e diferenciar os alunos que acertaram a questão dos que erraram. Pode-se observar que, mesmo os 9 alunos reprovando e refazendo a disciplina, houve uma 
Análise dos movimentos oculares durante a leitura de questões matemáticas por alunos de Engenharia que reprovaram em Cálculo Diferencial Integral
Maria Marilei Soistak Christo

Ângela Inês Klein

Luis Mauricio Martins de Resende

Romeu Miqueias Szmoski

Rafael Ribaski Borges

Dominik Grätz

pequena diminuição nos dados quantitativos analisados na tabela 2. A média de acertos da primeira vez que as questões foram resolvidas foi de $33 \%$, enquanto que na segunda resolução passou a 47\%, o que indicou que na segunda tentativa houve mais acertos que na primeira. O tempo médio de resolução diminuiu do primeiro para o segundo semestre, indo de 160 segundos para 108 segundos, o que indica que a compreensão do problema ocorreu mais rapidamente visto que faziam a disciplina pela segunda vez. A média do número de fixações e de sacadas teve também uma diminuição, o que se leva a inferir que, o fato de terem reprovado e refeito a disciplina proporcionou compreensão do problema com menor número médio de sacadas (394 na primeira resolução e 373 na segunda resolução) e fixações (383 na primeira resolução e 328 na segunda resolução).

Em relação a esta pesquisa realizada com alunos que reprovaram na disciplina é possível inferir que na segunda etapa os alunos estavam mais familiarizados com os termos matemáticos presentes nos problemas, porém, o significado e interpretação destes ainda não estavam claros e, portanto, a dificuldade na disciplina continua mesmo após a reprovação e o cursar novamente. Assim, é preciso investir em novas estratégias de melhorias do desenvolvimento da disciplina, para fins de diminuir a quantidade de reprovação e a dificuldade encontrada pelos alunos. A instituição do pré-cálculo com a retomada de conteúdos básicos necessários para o acompanhamento na disciplina de Cálculo Diferencial Integral I pode ser uma estratégia interessante, além de metodologias diferenciadas propostas pelos professores da área matemática e disciplinas correspondentes.

\section{Referências}

BARRETO, Ana Margarida. Eye tracking como método de investigação aplicado às ciências da comunicação. Revista Comunicando, v. 1, n. 1, p. 168-186, 2012.

CARPENTER, Patricia A.; JUST, Marcel A. Sentence comprehension: a psycholinguistic processing model of verification. Psychological Review, v. 82, n. 1, p. 45, 1975. https://doi.org/10.1037/h0076248

CHRISTO, Maria Marilei Soistak. Análise de movimentos oculares em questões de cálculo: um estudo desenvolvido com alunos de cursos de engenharia. 2019. 196 f. Tese (Doutorado em Ensino de Ciência e Tecnologia) - Universidade Tecnológica Federal do Paraná, Ponta Grossa, 2019.

DUCHOWSKI, Andrew T. Eye Tracking Methodology: Theory and Practice. London: Springer, 2007.

FINDLAY, John; WALKER, Robin. Human saccadic eye movements. Scholarpedia, v. 7, n. 7, 2012. https://doi.org/10.4249/scholarpedia.5095 
Análise dos movimentos oculares durante a leitura de questões matemáticas por alunos de Engenharia que reprovaram em Cálculo Diferencial Integral
Maria Marilei Soistak Christo

Ângela Inês Klein

Luis Mauricio Martins de Resende

Romeu Miqueias Szmoski

Rafael Ribaski Borges

Dominik Grätz

FORSTER, Renê. Aspectos da utilização do rastreamento ocular na pesquisa psicolinguística. DELTA: Documentação e Estudos em Linguística Teórica e Aplicada, v. 33, n. 2, 2017. https://doi.org/10.1590/0102-445095461720767529

GARZELLA, Fabiana Aurora Colombo. A disciplina de Cálculo I: a análise das relações entre as práticas pedagógicas do professor e seus impactos nos alunos. 2013. 298f. Tese (Doutorado em Educação) - Universidade Estadual de Campinas. São Paulo. 2013.

INHOFF, Albrecht Werner; RAYNER, Keith. Parafoveal word processing during eye fixations in reading: Effects of word frequency. Perception \& Psychophysics, v. 40, n. 6, p. 431-439, 1986. https://doi.org/10.3758/BF03208203

MACEDO, Eliseu Coutinho de et al. Processos perceptuais e cognitivos na leitura de palavras: propriedades dos movimentos oculares. Psicologia Escolar e Educacional, v. 11, n. 2, p. 275283, 2007. https://doi.org/10.1590/S1413-85572007000200007

OLIVEIRA, Maria Cristina Araújo; RAAD, Marcos Ribeiro. A existência de uma cultura escolar de reprovação no ensino de Cálculo. Boletim GEPEM, n. 61, p. 125-137, jul-dez. 2012. https://doi.org/10.4322/gepem.2014.018

RAYNER, Keith. Eye movements in reading and information processing: 20 years of research. Psychological Bulletin, v. 124, n. 3, p. 372, 1998. https://doi.org/10.1037/0033$\underline{2909.124 .3 .372}$

RAYNER, Keith; DUFFY, Susan A. Lexical complexity and fixation times in reading: Effects of word frequency, verb complexity, and lexical ambiguity. Memory \& Cognition, v. 14, n. 3, p. 191-201, 1986. https://doi.org/10.3758/BF03197692

REHFELDT, Márcia Jussara Hepp et al. Investigando os conhecimentos prévios dos alunos de Cálculo do Centro Universitário Univates. Revista de Ensino de Engenharia, v.31, n.1, p.2430, 2012. https://doi.org/10.15552/2236-0158/abenge.v31n1p24-30

UTFPR - UNIVERSIDADE TECNOLOGICA FEDERAL DO PARANÁ. Relatório de Gestão do exercício de 2018. Curitiba: EDUTFPR, 2019. Disponível em: http://portal.utfpr.edu.br/documentos/reitoria/documentos-institucionais/prestacao-decontas/2018-relatorio-de-gestao/view. Acesso em 4 de maio 2019.

ZARPELON, Edinéia. Análise do desempenho de alunos calouros de engenharia na disciplina de Cálculo Diferencial e Integral I: um estudo de caso na UTFPR. 2016. 120f. Dissertação (Mestrado em Ensino de Ciência e Tecnologia) - Universidade Tecnológica Federal do Paraná. Ponta Grossa. 2016. https://doi.org/10.26514/inter.v8i22.1416

ZARPELON, Edinéia; RESENDE, Luis Maurício Martins; REIS, Ednei Félix. Análise do desempenho de alunos ingressantes de engenharia na disciplina de Cálculo Diferencial e Integral I. Interfaces da educação, v. 8, n. 22, p. 305-335, 2017.

Recebido em: 04/10/2019. Aceito em: 30/12/2019. 